\title{
The Role of Psychosocial Factors in Functional Gastrointestinal Disorders
}

\author{
Jeffrey M. Lackner \\ Division of Gastroenterology, Department of Medicine, University at Buffalo School of Medicine, SUNY, \\ Buffalo, N.Y., USA
}

\begin{abstract}
Lacking a biomarker that reliably corresponds with Gl symptoms, functional GI disorders (FGIDs) are best understood from the perspective of a biopsychosocial model that emphasizes the reciprocal and interactive relationship among biological, environmental, and psychological factors. In more severe patients, psychological factors exert a particularly strong influence on the expression of $\mathrm{Gl}$ symptoms and related distress. This chapter describes 3 different pathways that link psychological processes to FGIDs with a focus on irritable bowel syndrome: psychological processes as vulnerability factors (early life adversity, neuroticism, observational learning) that increase the risk of developing FGIDs; psychological factors as determinants of illness behaviors that aggravate the day-to-day burden of FGIDs; psychological factors that directly impact biological processes (e.g. motility) that influence core symptoms of FGIDs, and psychological processes that, if targeted through evidence-based treatments (e.g. cognitive behavior therapy), have potential to relieve physical symptoms of FGIDs unresponsive to conventional medical or dietary treatments for more severely affected patients. This paper will describe these pathways with a focus on the FGIDs about which most is known (irritable bowel syndrome).
\end{abstract}

๑) 2014 S. Karger AG, Basel

Epidemiological data suggest that functional gastrointestinal disorders (FGIDs) are common and oftentimes disabling [1]. Irritable bowel syndrome (IBS) - the most common of FGIDs - affects $15 \%$ of the world's population at any given time. The addition of functional dyspepsia, chronic constipation, gastroesophageal reflux disease, and noncardiac chest pain makes FGIDs a public health problem of staggering proportions. Even though a small proportion of individuals with FGIDs seek medical care $[2,3]$, they account for $40-60 \%$ of all outpatient visits to GI clinics. Because they have no known etiology and their symptoms oftentimes mimic those of 'organic' diseases with underlying physical pathology, FGIDs are a source of frustration for physicians and of despair for patients, their families, and friends. Moreover, the costs - 
direct and indirect - associated with FGIDs impose a significant burden on our health care systems.

Lacking a reliable biomarker, FGIDs are therefore best understood from a biopsychosocial perspective [4]. The biopsychosocial model of FGIDs holds that individual biology (e.g. genetic predisposition, GI physiology), behavior, and higher-order cognitive processes (e.g. coping, illness beliefs, abnormal central processing of gut stimuli) influence FGIDs through their interaction with each other, with early-life factors (e.g. trauma, negative learning experiences), and with the individual's social and physical environments (e.g. reinforcement contingencies, interpersonal stress). At the heart of the model is recognition that FGIDs involve a dysregulation in interactions among the cognitive and emotional centers of the central nervous system (CNS), the immune system, the neuroendocrine system, and the enteric nervous system. Although alterations at any level of the neuroenteric axis may result in hallmark features of symptoms of FGIDs, multiple lines of evidence underscore the importance of CNS activity in modulating symptoms, particularly in more severely affected patients seen in tertiary care settings. Research has pointed to four main pathways through which psychological factors influence GI symptoms of FGIDs. This chapter will describe these pathways with a focus on the FGIDs about which most is known (IBS). An excellent source of information regarding psychosocial factors associated with functional dyspepsia can be found elsewhere [5].

\section{Psychological Factors Shape Physiological Responses}

The first key pathway linking psychological factors and FGIDs is directly through physiological systems that mediate gut sensation and function. A full review of these data is beyond the scope of this chapter, but the reader is encouraged to refer to Chang [6] for an excellent review of the literature. One of the earliest and most dramatic examples comes from Almy and colleagues whose laboratory studies in the 1940s and 1950s were designed to clarify the impact of stress on colonic function. In one study (1951), a healthy, 22-year-old medical student underwent a protoscopy which presumably detected 'carcinoma of the rectum'. The investigators requested the student to sign a consent permitting them to perform a biopsy. During the period when the biopsy was presumably performed, the student demonstrated heightened reactivity of gut function as measured by vascular engorgement of the bowel and increased contractions of gut above and beyond what was observed during a 10-min baseline period. After the student was debriefed and informed that information about the discovery of carcinoma was a hoax, colonic motility and engorgement were reevaluated and found to return to baseline levels. In other words, gut reactivity corresponded with the presentation (and termination) of psychological stress. These data led Almy to conclude that the colonic response depends not only upon the stimulus but also upon the subject's interpretation of the stimulus [7]. 
While stress-induced change in GI function is universal across both healthy individuals and functional GI patients, they are exaggerated in patients with FGIDs on core pathophysiological dimensions (e.g. gut motility, visceral perception) $[8,9]$. In one study linking laboratory induction of negative mood to increased colonic motility [8], IBS patients and controls underwent ambulatory motility recordings in the colon. Individuals in both groups were confronted on their return to the lab ('you're late', 'you came to the wrong window', 'now the study may need to be repeated') in a hostile manner that was designed to induce stress reactivity. Colonic motility increased during confrontation in the IBS patients, but not in healthy volunteers. A variety of psychological stressors (e.g. dichotomous listening tasks, driving in traffic, balls sorting) can elicit similar changes in GI motor function.

With respect to the effect of stress on visceral perception, there is some evidence that IBS patients report enhanced perceptual sensitivity when exposed to psychological stressors in laboratory settings. Dickhaus et al. [10], for example, reported that IBS patients reported significantly higher ratings of pain sensation and unpleasantness than controls following a rectal distension task while undergoing a psychologically stressful dichotomous listening tasks (i.e. different auditory inputs are played into the subjects' ears than controls). Similar findings have been reported by other researchers who have found that pain ratings fluctuate with the presentation of laboratory-induced psychological challenge [e.g. 11]. Other researchers have failed to find an effect of acute laboratory stressors on perception of colorectal distension. Inconsistent findings may be due to methodological differences in the design of studies. Even so, the

picture that emerges from broad review of data is that psychological factors (e.g. stress, strong negative emotions) directly affect physiological systems underlying gut sensation and function. These conclusions dovetail with what IBS patients report about the relationship between stress and GI symptoms. IBS patients report that psychological stress more frequently affects pain and bowel patterns than control subjects. Further, IBS patients report more stressful events, more stress-related changes in stool patterns, and greater reactivity to stress than controls [12]. Creed et al. [13] found that major life events preceded the onset of IBS in $60-66 \%$ of IBS patients.

\section{Psychological Factors Influence Illness Behaviors}

A second pathway through which psychological factors influence IBS symptoms is the adoption of illness behaviors. The term 'illness behaviors' refers to maladaptive ways of responding to one's illness [14]. Illness behaviors can take one of two extreme forms [15]. At one end, an individual may fail to detect and act on troublesome somatic sensations that are amenable to medical treatment. An example is an elderly individual who does not seek appropriate care for chronic constipation because of the mistaken belief that it is a normative and uncontrollable part of the aging process for which there are no helpful treatments. At the other extreme are individuals who are preoccupied 
with fears of having a serious disease based on the person's interpretation of body sensations. These fears are behaviorally expressed in various forms such as resting in bed, taking excessive medications, urging doctors to perform diagnostic tests of limited value, 'doctor shopping', exhibiting facial expression of discomfort or verbal complaints, or engaging in avoidance behaviors. The most overt forms of avoidance include refusal to enter situations where symptoms are expected to occur (e.g. avoiding shopping in shopping malls where the location of bathrooms is unknown) and escaping from unpleasant situations shortly after entering (e.g. leaving a party after $15 \mathrm{~min}$ because of fear that low level of nausea will worsen and cause a loss of control).

In addition to overt behaviors, FGID patients can engage in more subtle 'safety behaviors' that are used to protect themselves from feeling overly uncomfortable or to prevent their undesirable consequences (e.g. loss of bowel control, embarrassment, pain) from actually occurring. Patients who engage in safety behaviors do not necessarily outright avoid a situation or activities but will only do so if they take certain precautions. For example, someone who is fearful of losing control of bowel function may only feel comfortable going out to dinner if she carries a bottle of Imodium or an extra pair of underwear in her purse. In this case, the bottle of Imodium becomes a 'safety signal'. Safety signals are regarded as more subtle forms of avoidance because they are believed to help the person escape or avoid a negative outcome. The individual assumes that managing symptoms depends on the safety signal, and the aversive situation is manageable only when the safety signal is present. Safety behaviors reinforce the assumption that the situation would be intolerable if the safety signal was absent. Engaging in safety behaviors prevents disconfirmation of threat appraisals, thereby heightening anxiety which can aggravate somatic symptoms. In the long term, excessive use of safety behaviors increases anxiety and self-focused attention and causes a misattribution of safety. That is, the patient who relies on safety behaviors to cope with IBS flare-ups attributes their nonoccurrence to a safety behavior not to his/ her own coping behavior.

\section{Patient Beliefs}

Illness behaviors are influenced by how patients describe aspects of their illness. Patients vary in how they interpret their symptoms as well as the intensity, usefulness, and valence (positive vs. negative) of these beliefs, expectations, and other thoughts. Negatively skewed thinking patterns are strong determinants of health outcomes such as pain, function, and quality of life. One overly negative belief that influences illness behavior is called catastrophizing [16]. Catastrophizing is characterized by a tendency to exaggerate the threat value of aversive symptoms [12] such as pain, diarrhea, or urgency. Catastrophizing comprises 3 different cognitive elements: rumination (e.g. 'I can't stop thinking about how bad it would be'), magnification ('I worry that something serious may happen'), and helplessness ('There is nothing I can do to 
control it'). Catastrophizing has been most extensively studied in the context of chronic pain patients who tend to distort their evaluations of their own behavior and performance in an overly negative manner [17]. Sullivan and Neish [18] showed that catastrophizing scores predict pain and functional status (e.g. perceived disability, employment status). Drossman et al. [19] were one of the first research teams to extend the construct of catastrophizing to patients with FGIDs. They measured catastrophizing in patients with IBS, and found that it helped predict subsequent (12-month) health outcomes as measured by a composite health status of six variables (current pain, bed disability days, psychological distress, daily dysfunction, number of physician visits, number of surgeries and procedures) that was heavily weighted toward illness behaviors.

Another cognitive information processing variable that influences treatment seeking behavior are patients' worries. Worry is distinguished from pain catastrophizing on the basis of its uncontrollability, narrative quality, inherent future orientation, chronicity, and pervasiveness (i.e. problem topics are not specific to a single situation or stimuli). Whereas the focal problem underlying pain catastrophizing is by definition relatively well defined, worry is abstract, conceptual, and nonconcrete [20]. Unlike pain catastrophizing, worry functions as a way of avoiding perceived threat [21] that does not physically exist in the 'here and now' and for which there is no available behavioral response (e.g. escape). Worrying distracts from emotional distress and suppresses unpleasant somatic sensations in a manner that is negatively reinforcing and lends uncontrollability to the worry process. Difficulty controlling worry often results in prolonged bouts of worry that are difficult to 'turn off, which may cause significant distress and functional impairment. High levels of worry are associated with heightened focus on bodily symptoms, repeated checking for signs and symptoms related to health concerns, focus on the negative consequences of physical sensations, and frequent efforts to obtain reassurance from family members, friends, or health care professionals. FGID patients who worry that their GI symptoms (e.g. abdominal pain) signify an underlying physical disease (e.g. colon cancer) are more heavily represented among consulters than nonconsulters [22]. Gomborone et al. [23] compared illness attitudes (e.g. health worries) between patients with IBS and organic GI disease, and found that the IBS patients reported more health worries. The magnitude of health worry was not explained by the severity of either coexisting mental distress or somatic symptoms.

Levels of coexisting emotional distress can also influence the expression of illness behaviors [24]. Multiple studies of FGID patients recruited from tertiary care settings indicate that they are characterized by relatively high levels of anxiety, depression, or other forms of distress compared to nonconsulters [25]. Whitehead et al. [26] showed that treatment-seeking IBS patients had higher scores than normal subjects and nonconsulting individuals with IBS symptoms on multiple dimensions (anxiety, somatization, interpersonal sensitivity, etc.) of a psychiatric symptom checklist. Similar findings were reported by Drossman et al. [27]. Individuals with IBS symptoms who do 
not seek medical care for IBS symptoms generally have a normal psychological profile, although some studies show that their levels of psychological distress fall within intermediate levels (i.e. between normal and clinical ranges $[28,29]$ ).

\section{Psychiatric Comorbidity in IBD and Functional Gastrointestinal Disorders}

Other researchers have approached the issue of psychiatric comorbidity by examining the proportion of FGID patients who meet diagnostic criteria for a formal mental disorder. Psychiatric comorbidity is relatively commonplace among FGID patients presenting at tertiary clinics. In studies [30] that have administered structured clinical interviews to establish the rate of psychiatric comorbidity among IBS patients per criteria as specified in the American Psychiatric Association's Diagnostic and Statistical Manual of Mental Disorders [31], approximately 60\% of treatmentseeking IBS patients have a diagnosable psychiatric condition [32]. By comparison, approximately $25 \%$ of American adults suffer from a diagnosable mental disorder $[33,34]$.

The most frequent class of psychiatric diagnoses are the anxiety disorders. Within this class, the most prevalent anxiety disorders is generalized anxiety disorder (GAD) whose key defining feature is chronic feelings of intense (uncontrollable, excessive) worry concerning a range of different topics. For the individual with GAD, the target of worry is not limited to a single topic such as health concerns. The focus of worry may involve a generalized variety of daily life events due to (a) a sense that he/she is often unable to cope with those events or reactions to events; (b) fear of making mistakes or failing in any task of importance; and (c) concern with being negatively evaluated by oneself and by others. Intense worry is often accompanied by nervousness, physical tension, restlessness, and other symptoms of CNS arousal.

In addition to anxiety disorders, a significant proportion of IBS patients meet diagnostic criteria for a mood disorder. Between 12 and 28\% meet criteria for major depressive disorder and 7\% dysthymic disorder [35-37]. It is believed that negative mood states arising from psychiatric comorbidities may negatively bias interpretations of symptoms, which leads to increased symptom reporting and health care seeking [24]. Research that has attempted to identify the temporal relationship between onset of IBS and comorbid psychiatric diagnoses has found that mood disorders appear to precede the onset of IBS, whereas anxiety disorders typically follow IBS onset [38]. Other psychiatric comorbidities include somatoform disorders, a group of disorders that cause medically unexplained somatic symptoms [39].

Of course, even if up to $60 \%$ of treatment-seeking IBS patients have a psychiatric diagnosis, a substantial proportion (40\%) do not have a formal mental illness. This means that psychiatric comorbidity alone is insufficient for fully understanding the contribution of psychological factors to FGIDs. This has prompted some researchers to shift the focus from distress emanating from comorbid psychiatric conditions to 
distress arising from GI-specific symptoms [40]. To this end, UCLA researchers have focused on the concept known as GI-specific anxiety. GI-specific anxiety refers to a fear of visceral sensations ('I often fear that I won't be able to have a normal bowel movement') and their consequences ('I get anxious when I go to a new restaurant') [41]. Situations that are likely to trigger GI symptom-specific anxiety include those involving food and eating such as restaurants and parties or locations where the availability of bathroom facilities is unknown. GI-specific anxiety is conceived as a marker of overresponsiveness to GI sensations which is believed to perpetuate IBS symptoms through alterations in autonomic and pain facilitation, as well as cognitive (e.g. attention, internal self-focus) mechanisms [41]. GI-specific anxiety may be associated with beliefs of poor symptom control and high illness impact and may, therefore, be a critical element in the severe decrements in quality of life found in IBS patients. GIspecific anxiety has been shown to be significantly associated with IBS severity and impact even after controlling for trait anxiety. GI symptom-specific anxiety is regarded as an especially important psychosocial variable underlying visceral pain sensitivity, hypervigilance, and maladaptive coping [40, 42]. It is unclear whether levels of visceral anxiety influence health care seeking.

\section{Psychological Processes as Psychological Vulnerability Factors}

A third pathway through which psychological factors influence IBS is by increasing the risk of developing IBS. In general, this line of research has focused on 3 types of psychosocial risk factors - negative learning experiences, personality traits, and life stress (trauma, stressors) [43]. One vulnerability factor may be a self-reported history of early-life adversity (e.g. physical and or sexual abuse).

\section{Trauma History}

A history of sexual abuse (as defined by nonconsensual sexual contact obtained through unwanted touching/fondling and physical contact with the sexual parts of the victim's or the offender's body through force or threat of harm or rape) and/or physical trauma (i.e. frequent beating, life threat) occurs in $20-60 \%$ of IBS patients seen in the US and Europe [44]. Drossman et al. [45] found that the frequency of a history of abuse among functional GI disorder patients was 30\% greater than in women with an organic GI disease. Early-life adversity is believed to induce changes in the CNS, including altered function of the hypothalamic-pituitary-adrenal axis and the sympathetic nervous system, in genetically predisposed individuals [46, 47]. Additionally, trauma may cause epigenetic programming of glucocorticoid receptor expression that affects behavioral adaption to vulnerability to stress-related disorders. Information regarding abuse history is clinically important for a cohort of 
patients because it corresponds with refractory symptoms, greater healthcare utilization (e.g. more frequent visits to the doctor), and susceptibility to undergoing unneeded medical procedures $[45,48]$. The high frequency of abuse is, however, not specific to IBS patients and is reported by patients with other persistent pain disorders such as fibromyalgia, headache or pelvic pain. In this respect, trauma is not a cause of IBS. At best, it can be understood as an early stressor that occurs with relatively high frequency and may predispose some individuals to adult-onset IBS and poorer outcomes.

Additional evidence supporting the role of a stress as a risk factor for IBS comes from studies [49] of patients who develop IBS after a bout of infectious gastroenteritis (i.e. postinfectious IBS or PI-IBS). Approximately $10 \%$ of patients with PI-IBS retrospectively attribute the onset of symptoms to an infectious illness [50]. While most patients rapidly recover from bacterial gastroenteritis, up to a third of patients experience persistent GI symptoms with a portion of those affected meeting diagnostic criteria for IBS [51]. Risk factors for developing PI-IBS include, in descending order of importance: duration of initial illness, toxicity of infecting bacterial strain, smoking, mucosal markers of inflammation, female gender, depression, hypochondriasis, and the occurrence of stressful life events (particularly interpersonal problems such as relationship breakup, loss) within 3 months of the infectious illness. Of 8 risk factors for onset of PI-IBS, 4 are psychosocial factors. These data are consistent with the work of Dunlop et al. [49] who found that the power of depression scores to independently predict PI-IBS rivaled rectal mucosal enterochromaffin cell count, suggesting that both biological and psychological factors are important in the development of PI-IBS. The underlying mechanisms whereby psychological factors increase the risk of PI-IBS are unclear, but one possibility is that they maintain low-grade inflammation.

\section{Neuroticism}

A number of researchers have focused attention on personality traits as risk factors for IBS. While there is no evidence of a unique IBS-prone personality, a proportion of individuals have a temperamental vulnerability that puts them at risk for developing IBS symptoms. The temperamental vulnerability that is most often linked with IBS is neuroticism (also referred to as negative emotionality or negative temperament). Neuroticism reflects a broad and pervasive personality dimension that helps explain individual differences in the propensity to experience negative emotions including sadness, fear, anxiety, anger, frustration, as well as associated characteristics including low self-esteem, worry and insecurity.

Neuroticism is associated with a tendency to experience negative affect in the face of minor stressors, to experience arousal quickly and difficulty returning to baseline following a period of evoked response. Physiologically, elevated neuroticism is associated with increased autonomic and neural responses to negative stimuli [52-54]. 
Cognitively, persons with high scores on neuroticism scales tend to have an internal self-focus (i.e. somatically focused) and a propensity to interpret noxious bodily sensations in a negative manner. On self-report measure, high neuroticism individuals report more bothersome symptoms, interpret their symptoms as more severe and express more general emotional distress than low neuroticism individuals. It is not surprising then that neuroticism has emerged as a psychological risk factor for a variety of emotional disorders and physical conditions [55].

In the case of FGIDs, a number of studies have documented the link between neuroticism and IBS $[29,56]$. High scores on neuroticism scales characterize individuals with IBS symptoms [57], and neuroticism is regarded as a risk factor for chronic unexplained pain in IBS. Neuroticism may influence IBS experience by lowering the sensitivity threshold at which noxious symptoms (e.g. pain) are perceived as threatening, intolerable, and requiring medical intervention. Consistent with this hypothesis are findings from a study [58] comparing personality style of normal individuals, lactose malabsorbers (LMAs), and individuals with IBS in the general community who do not seek medical attention, versus LMAs and individuals with IBS who do seek healthcare. Higher rates of neuroticism characterized both LMAs and patients with IBS who sought medical assistance. The precise mechanisms underlying the effects of neuroticism on treatment seeking and other illness behaviors of IBS patients are not well established. Candidate explanations include increased awareness of somatic sensations, catastrophic appraisal of benign sensations as dangerous, and corresponding lower threshold for seeking medical care. Other researchers have argued that neuroticism confers a proneness to distress that can aggravate somatic sensations [59].

\section{Observational Learning}

Working from a social learning theory (SLT) perspective [60], Whitehead et al. [61] emphasize negative learning experiences in the etiology of FGIDs. SLT is the prevailing theoretical framework used in health behavior and health promotion research. In SLT, human behavior is explained in terms of a three-way, dynamic, reciprocal theory in which individual (e.g. biology, emotions, cognitions) factors, environmental influences, and behavior interact continually. A basic premise of SLT is that people learn not only through their own experiences but also by observing the actions of others (i.e. observational learning, modeling) and the results of those actions (consequences). The family environment is a fertile context in which learning occurs. Whitehead et al. [62] contend that complaints of IBS symptoms are partly acquired by children who learn through direct experience and by observing how others (e.g. parents) respond to IBS. In support of the general principle of familial transmission are results of a large telephone survey that found that individuals who reported IBS-like symptoms were likely to recall that their parents both responded to GI 
symptoms such as stomach aches with the provision of positive reinforcement (e.g. gifts, toys) and demonstrated illness behaviors (school absences, physician visits) that can negatively reinforce symptoms. Similarly, Levy et al. [63] found that children of IBS parents sought medical care for GI and non-GI complaints more than matched controls. These data are consistent with the notion that experience of IBS is to some extent shaped by negative learning experiences formed in the family environment [64].

\section{Psychological Treatments as Evidence-Based Treatments}

The fourth pathway linking psychological factors to IBS is through the application of psychological techniques to relieve IBS symptoms and related distress. In essence, psychological treatments are specifically designed to alter psychological processes thought to underlie or significantly contribute to pain, distress, and disability or normalize gut function. The most well-studied psychological treatment is a specific therapy called cognitive behavior therapy or CBT. The cognitive part of CBT helps people change specific thinking patterns that heighten physiological and psychological arousal, while the behavioral part helps people change the way they react to situational triggers. CBT techniques (e.g. cognitive restructuring, relaxation techniques) have been administered either singularly or in combination with other interventions and have been featured in over 30 randomized clinical trials since 1985. CBT generally yields broad improvements in GI symptoms (pain, bowel dysfunction) and quality of life. Research summarized in a recent systematic review suggests that psychological treatments as a whole are at least moderately effective in reducing IBS symptoms $[65,66]$. Whether treatment gains following a regimen of CBT are enduring in the longer term is unclear. Although there were not enough data to establish the relative superiority of any one type of psychological treatment, most trials featured CBT. A recent study using CBT to treat severely affected IBS patients found that the great majority of patients reported significant reduction in the severity of IBS symptoms and reported adequate relief from IBS symptoms [67]. The therapeutic value of CBT was echoed in a recently published New England Journal of Medicine [68] narrative review that identified $\mathrm{CBT}$ as one of the few empirically validated treatments for IBS. Other evidence-based psychological treatments include hypnosis and interpersonal psychotherapy [69].

\section{Acknowledgements}

This study was supported in part by a research grant from the National Institutes of Health, NIDDK (DK77738). 


\section{References}

1 Chang L: Review article: epidemiology and quality of life in functional gastrointestinal disorders. Aliment Pharmacol Ther 2004;20(suppl 7):31-39.

2 Drossman DA, Sandler RS, McKee DC, et al: Bowel patterns among subjects not seeking health care. Use of a questionnaire to identify a population with bowel dysfunction. Gastroenterology 1982;83:529_ 534.

-3 Thompson WG, Heaton KW: Functional bowel disorders in apparently healthy people. Gastroenterology 1980;79:283-288.

4 Drossman DA: Presidential address: gastrointestinal illness and the biopsychosocial model. Psychosom Med 1997;60:258-267.

5 Van Oudenhove L, Aziz Q: The role of psychosocial factors and psychiatric disorders in functional dyspepsia. Nat Rev Gastroenterol Hepatol 2013;10:158167.

6 Chang L: The role of stress on physiologic responses and clinical symptoms in irritable bowel syndrome. Gastroenterology 2011;140:761-765 e5.

7 Almy TP, Kern F, Tulin M: Alterations in colonic function in man under stress. II. Experimental production of sigmoid spasm in healthy persons. Gastroenterology 1949;12:425-436.

8 Welgan P, Meshkinpour H, Beeler M: Effect of anger on colon motor and myoelectric activity in irritable bowel syndrome. Gastroenterology 1988;94:11501156.

9 Welgan P, Meshkinpour H, Hoehler F: The effect of stress on colon motor and electrical activity in irritable bowel syndrome. Psychosom Med 1985;47: 139-149.

10 Dickhaus B, Mayer EA, Firooz N, et al: Irritable bowel syndrome patients show enhanced modulation of visceral perception by auditory stress. Am J Gastroenterol 2003;98:135-143.

11 Murray CDR, Flynn J, Ratcliffe L, et al: Effect of acute physical and psychological stress on gut autonomic innervation in irritable bowel syndrome. Gastroenterology 2004;127:1695-1703.

12 Bennet EJ, Tennant CC, Piesse C, et al: Level of chronic life stress predicts clinical outcome in irritable bowel syndrome. Gut 1998;43:256-261.

13 Creed F, Craig T, Farmer R: Functional abdominal pain, psychiatric illness, and life events. Gut 1988;29: 235-242.

14 Pilowsky I: The diagnosis of abnormal illness behaviour. Aust N Z J Psychiatry 1971;5:136-138.

15 Levy RL, Whitehead WE, Von Korff MR, et al: Intergenerational transmission of gastrointestinal illness behavior. Am J Gastroenterol 2000;95:451456.
16 Turner JA, Aaron LA: Pain-related catastrophizing: what is it? Clin J Pain 2001;17:65-71.

17 Lefebvre MF: Cognitive distortion and cognitive errors in depressed psychiatric and low back pain patients. J Consult Clin Psychol 1981;49:517-525.

18 Sullivan M, Neish NR: Catastrophizing, anxiety and pain during dental hygiene treatment. Community Dent Oral Epidemiol 1998;26:344-349.

19 Drossman DA, Li Z, Leserman J, et al: Effects of coping on health outcome among female patients with gastrointestinal disorders. Psychosom Med 2000;62: 309-317.

20 Kindt M, Brosschot JF, Boiten F: High-Level cognition in phobics: abstract anticipatory memory is associated with the attenuation of physiological reactivity to threat. J Anxiety Disord 1999;13:473-489.

21 Borkovec TD, Hu S: The effect of worry on cardiovascular response to phobic imagery. Behav Res Ther 1990;28:69-73.

22 Creed FH, Levy RL, Bradley LA, et al (eds): Psychosocial Aspects of Functional Gastrointestinal Disorders. McLean, Degnon Associates, 2006.

23 Gomborone J, Dewsnap P, Libby G, et al: Abnormal illness attitudes in patients with irritable bowel syndrome. J Psychosom Res 1995;39:227-230.

24 Cohen S, Rodriguez M: Pathways linking affective disturbances and physical disorders. Health Psychol 1995;14:374-380.

25 Koloski NA, Talley NJ, Boyce PM: Epidemiology and health care seeking in the functional GI disorders: a population-based study. Am J Gastroenterol 2002; 97:2290-2299.

26 Whitehead WE, Bosmajian L, Zonderman AB, et al: Symptoms of psychologic distress associated with irritable bowel syndrome. Comparison of community and medical clinic samples. Gastroenterology 1988; 95:709-714.

27 Drossman DA, McKee DC, Sandler RS, et al: Psychosocial factors in the irritable bowel syndrome. A multivariate study of patients and nonpatients with irritable bowel syndrome. Gastroenterology 1988;95: 701-708.

28 Mertz H: Role of the brain and sensory pathways in gastrointestinal sensory disorders in humans. Gut 2002;51(suppl 1):i29-i33.

29 Talley NJ, Boyce PM, Jones M: Is the association between irritable bowel syndrome and abuse explained by neuroticism? A population based study. Gut 1998; 42:47-53.

30 Blanchard EB: Irritable Bowel Syndrome: Psychosocial Assessment and Treatment. Washington, American Psychiatric Association, 2000. 
31 American Psychiatric Association: Diagnostic and Statistical Manual of Mental Disorders, ed 4. Washington, American Psychiatric Association, 1994.

32 Blanchard EB, Scharff L: Psychosocial aspects of assessment and treatment of irritable bowel syndrome in adults and recurrent abdominal pain in children. J Consult Clin Psychol 2002;70:725-738.

33 Alegria M, Jackson JS, Kessler RS, et al: National Comorbidity Survey Replication (NCS-R) 2001-2003. Ann Arbor, Inter-University Consortium for Political and Social Research, 2003.

34 Kessler RC, Berglund P, Chiu WT, et al: The US National Comorbidity Survey Replication (NCS-R): design and field procedures. Int J Methods Psychiatr Res 2004;13:69-92.

35 Lydiard RB, Fossey MD, Marsh W, et al: Prevalence of psychiatric disorders in patients with irritable bowel syndrome. Psychosomatics 1993;34:229234.

36 Canavan JB, Bennett K, Feely J, et al: Significant psychological morbidity occurs in irritable bowel syndrome: a case-control study using a pharmacy reimbursement database. Aliment Pharmacol Ther 2009; 29:440-449.

37 Ballenger JC, Davidson JR, Lecrubier Y, et al: Consensus statement on depression, anxiety, and functional gastrointestinal disorders. J Clin Psychiatry 2001;62(suppl 8):48-51.

38 Sykes MA, Blanchard EB, Lackner J, et al: Psychopathology in irritable bowel syndrome: support for a psychophysiological model. J Behav Med 2003;26: 361-372.

39 Miller AR, North CS, Clouse RE, et al: The association of irritable bowel syndrome and somatization disorder. Ann Clin Psychiatry 2001;13:25-30.

40 Labus JS, Mayer EA, Chang L, et al: The central role of gastrointestinal-specific anxiety in irritable bowel syndrome: further validation of the visceral sensitivity index. Psychosom Med 2007;69:89-98.

41 Labus JS, Mayer EA, Chang L, et al: The central role of gastrointestinal-specific anxiety in irritable bowel syndrome: further validation of the Visceral Sensitivity Index. Psychosom Med 2007;69: 89-98.

42 Labus JS, Bolus R, Chang L, et al: The Visceral Sensitivity Index: development and validation of a gastrointestinal symptom-specific anxiety scale. Aliment Pharmacol Ther 2004;20:89-97.

43 Drossman DA: Irritable bowel syndrome. Gastroenterologist 1994;2:315-326.

44 Drossman DA: Abuse, trauma, and GI illness: is there a link? Am J Gastroenterol 2011;106:14-25.

45 Drossman DA, Leserman J, Nachman G, et al: Sexual and physical abuse in women with functional or organic gastrointestinal disorders. Ann Intern Med 1990;113:828-833.
46 Chang L, Sundaresh S, Elliott J, et al: Dysregulation of the hypothalamic-pituitary-adrenal (HPA) axis in irritable bowel syndrome. Neurogastroenterol Motil 2009;21:149-159.

47 Videlock EJ, Adeyemo M, Licudine A, et al: Childhood trauma is associated with hypothalamic-pituitary-adrenal axis responsiveness in irritable bowel syndrome. Gastroenterology 2009;137:1954-1962.

48 Drossman DA, Talley N, Leserman J, et al: Sexual and physical abuse and gastrointestinal illness: review and recommendations. Ann Intern Med 1995; 123:782-789.

49 Dunlop SP, Jenkins D, Neal KR, et al: Relative importance of enterochromaffin cell hyperplasia, anxiety, and depression in postinfectious IBS. Gastroenterology 2003;125:1651-1659.

50 Collins SM, Vallance B, Barbara G, et al: Putative inflammatory and immunological mechanisms in functional bowel disorders. Baillieres Best Pract Res Clin Gastroenterol 1999;13:429-436.

51 Spiller R, Garsed K: Infection, inflammation, and the irritable bowel syndrome. Dig Liver Dis 2009;41: 844-849.

52 Coen SJ, Kano M, Farmer AD, et al: Neuroticism influences brain activity during the experience of visceral pain. Gastroenterology 2011;141:909917 e1.

53 Canli T: Functional brain mapping of extraversion and neuroticism: learning from individual differences in emotion processing. J Pers 2004;72:1105-1132.

54 Norris CJ, Larsen JT, Cacioppo JT: Neuroticism is associated with larger and more prolonged electrodermal responses to emotionally evocative pictures. Psychophysiology 2007;44:823-826.

55 Charles ST, Gatz M, Kato K, et al: Physical health 25 years later: the predictive ability of neuroticism. Health Psychol 2008;27:369-378.

56 Talley NJ, Boyce PM, Jones M: Predictors of health care seeking for irritable bowel syndrome: a population based study. Gut 1997;41:394-398.

57 Hazlett-Stevens H, Craske MG, Mayer EA, et al: Prevalence of irritable bowel syndrome among university students: the roles of worry, neuroticism, anxiety sensitivity and visceral anxiety. J Psychosom Res 2003;55:501-505.

58 Enck P, Holtkotter B, Whitehead W, et al: Clinical symptoms, psychopathology and intestinal motility in patients with 'irritable bowel' (in German). Z Gastroenterol 1989;27:357-361.

59 Wade JB, Price DD: Nonpathological factors in chronic pain: implications for assessment and treatment; in Gatchel RJ, Weisberg JN (eds): Personality Characteristics of Patients with Pain. Washington, American Psychological Association, 2000.

60 Bandura A: Self-efficacy: toward a unifying theory of behavioral change. Psychol Rev 1977;84:191-215. 
61 Whitehead WE, Bosmajian L, Zonderman AB, et al: Symptoms of psychologic distress associated with irritable bowel syndrome: comparison of community and medical clinical samples. Gastroenterology 1988;95:709-714.

62 Whitehead WE, Winget C, Fedoravicius AS, et al: Learned illness behavior in patients with irritable bowel syndrome and peptic ulcer. Dig Dis Sci 1982; 27:202-208.

63 Levy RL, Whitehead WE, Walker LS, et al: Increased somatic complaints and health-care utilization in children: effects of parent IBS status and parent response to gastrointestinal symptoms. Am J Gastroenterol 2004;99:2442-2451.

64 Chitkara DK, van Tilburg MAL, Blois-Martin N, et al: Early life risk factors that contribute to irritable bowel syndrome in adults: a systematic review. Am J Gastroenterol 2008;103:765-774.
65 Ford AC, Vandvik PO: Irritable bowel syndrome. Clin Evid (Online) 2012;2012:pii 0410.

66 Lackner JM, Morley S, Dowzer C, et al: Psychological treatments for irritable bowel syndrome: a systematic review and meta-analysis. J Consult Clin Psychol 2004;72:1100-1113.

67 Lackner JM, Jaccard J, Krasner SS, et al: Self-administered cognitive behavior therapy for moderate to severe irritable bowel syndrome: clinical efficacy, tolerability, feasibility. Clin Gastroenterol Hepatol 2008;6:899-906.

68 Mayer EA: Clinical practice. Irritable bowel syndrome. N Engl J Med 2008;358:1692-1699.

69 Naliboff B, Lackner JM, Mayer E: Psychosocial factors in the care of patients with functional gastrointestinal disorders; in Yamada T (ed): Principles of Clinical Gastroenterology. New York, John Wiley \& Sons, 2008, pp 20-37.

Jeffrey M. Lackner, Psy.D.

Behavioral Medicine Clinic, Department of Medicine, UB School of Medicine, SUNY

ECMC, 462 Grider Street

Buffalo, NY 14215 (USA)

E-Mail lackner@buffalo.edu 